\title{
ULTRASONIC NEURAL PROBE FOR REAL TIME ELECTROMECHANICAL HISTOLOGY OF NEURAL INTERFACES
}

\author{
P. C. Chen ${ }^{1 *}$, C. J. Shen ${ }^{1,2}$, J. C. Kuo ${ }^{1}$, C. G. Clark ${ }^{2}$, B. Nijjar ${ }^{2}$, C. Schaffer ${ }^{2}$, R.F. Gilmour, Jr. ${ }^{3}$, and A. Lal ${ }^{1,2}$ \\ ${ }^{1}$ SonicMEMS Laboratory, School of Electrical and Computer Engineering \\ ${ }^{2}$ Department of Biomedical Engineering, ${ }^{3}$ Department of Biomedical Sciences, \\ Cornell University, Ithaca, NY, USA
}

\begin{abstract}
We present a neural probe with first-ever capability to measure and alter the mechanical environment of the probe. The probe is a silicon catenoidal ultrasonic horn consisting of two platinum electrode recording sites, and four polysilicon resistors for a strain gauge. The strain gauge provides transverse displacement sensitivities of $0.043 \mathrm{~V} / \mathrm{m}$ and longitudinal force sensitivities of 20 $\mathrm{mV} / \mathrm{mN}$. A miniature recording ASIC system with $50 \mathrm{~dB}$ gain neural amplifier and radio frequency module, and instrument amplifier with gain of 200 is used to transmit the neural electrical and strain signals, providing simultaneous electrical and mechanical signals from the probes. A microcontroller is used to determine and transmit the probe resonance frequencies. Using ultrasonic actuation of the probe we demonstrate in vivo measurement of insertion force reduction by a factor of 2.3, average tissue-probe stress reduction by a factor of 1.5 ,as well as breathing induced micromotion and the probe resonance frequency. These data can be useful for characterizing and improving neural probe and tissue-electrical interface reliability.
\end{abstract}

\section{INTRODUCTION}

Neural interfaces can be used to record large-scale neuronal activity and the information extracted from these recordings can, in turn, be used to control prosthetics. Neural interface technology can provide a very promising solution to treating people with paralysis or lost limbs [1]. By implanting electrodes that can record and stimulate neural activity with good spatial and temporal resolution, these technologies can help bridge a patient's cortical activity to limbs or external devices. However, the reliability of the neural interfaces is a key challenge for chronic use of neural probes for medical treatments.

Many researchers have observed that the loss of electrode function after chronic insertion is reduced to a zero signal-to-noise ratio. Studies indicate two main types of reactions resulting from electrodes. The first results from the initial insertion trauma, which can involve severing of capillaries, the extracellular matrix, and cell processes [2]. It has been observed that the initial insertion trauma causes hemorrhage and edema, in addition, to a microglia response that can fade, in some cases, after a few weeks [2]. This phenomenon of an initial insertion trauma response is supported by control studies which examine the impact of electrode stab wounds on tissue. These studies demonstrate little trace of electrode tracks several months after the stab wounds. In addition, various studies have pointed to different foreign body response reactions to electrode insertion, depending on insertion material, tip-shape, insertion velocity, and other conditions. However, despite numerous studies, in practice, the ability of maintaining a good neural interface remains a black art.

As the immune reaction to probe insertion is caused by the presence of foreign material in the body, various strategies have been proposed and attempted to reduce the immune reaction and thereby increase the lifetime of the electrodes. The effect of silicon electrode tip shape has been investigated as a potential way to reduce damage to cells. It has been demonstrated that a chisel-point silicon electrode tip shape can produce a kill zone of less than $10 \mathrm{~m}$
[3]. Additionally, electrode insertion velocity may have an effect on tissue response. Strategies have been developed to allow insertion of 100 electrodes simultaneously while producing no significant long-term damage [4]. Thinner and less stiff materials are also being investigated to determine their effect on reducing tissue immune reactions. However, one of the challenges of using very thin, flexible materials is proper insertion of the electrodes into the areas of interest. While dissolvable silk with patterned thin electronics has been used for neural recordings, these films have only been placed on the surface of the cortex [5]. Biocompatible coating techniques to decrease electrode impedance also have been investigated. Conductive polymers can decrease the initial impedance of electrodes and reduce the electrode size required to record from a given area [6]. Additionally, conductive polymer coatings, such as Poly (3,4-ethylenedioxythiophene) (PEDOT) can increase the charge injection capability of a material [7]. When inserted for long-term neural recordings, PEDOT coated electrodes exhibit lower electrode site impedance versus uncoated electrodes for a period of at least 40 days post surgery, after an initial swelling period disappears [8].

There are many challenges in terms of achieving repeatable reactions to inserted electrodes and creating viable solutions to allow decades long high quality electrical recordings from neural interfaces. However, by developing quantitative methods to assess tissue-electrode reactions in vivo and ex vivo in large numbers of animals, we can seek to better understand the problem. In this paper we use ultrasonically actuated neural probes to address reliability. We report on the first-ever multifunctional ultrasonic neural probe that can be actuated ultrasonically during insertion, greatly reducing the insertion force and reducing the average stress around the probe (Fig. 1a, b). Furthermore, the probe can measure viscoelastic properties of the probe-tissue interface and micromotion can be monitored via measuring the ultrasonic response of the probes (Fig. 1c, d). We provide measurements in mice for in situ probe histology. It is hypothesized that the reactive tissue response can be decreased by reducing the insertion force and possibly even eliminated by periodically actuating the probe with ultrasound.

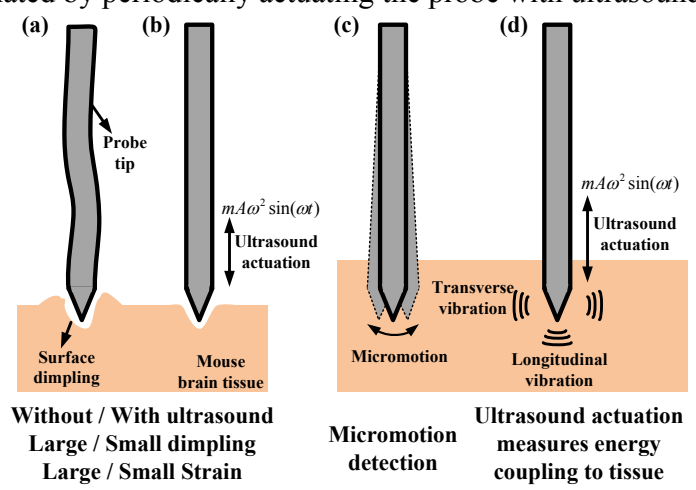

Figure 1: Probe insertion without (a) and with (b) ultrasonic actuation. Applying the ultrasonic driving voltage reduces dimpling and insertion force. (c)Micromotion detection (d) Energy coupling of the probe-tissue interface can be monitored via the ultrasonic response (longitudinal/transverse) of the probes. 


\section{PROBE PROCESS}

The silicon neural probes are designed in a catenoidal horn shape (Fig. 2) [9] for maximum tip motion, with a resonance frequency of $430 \mathrm{kHz}$. Probes are fabricated using a similar process to [9] from $<100>$ silicon wafer. Briefly, polysilicon strain gauge resistors are realized by LPCVD polysilicon implanted with boron at a dose of $2 * 10^{15}$ ions $/ \mathrm{cm}^{2}$ at $100 \mathrm{keV}$. The resistors are electrically contacted with aluminum alloy $(\mathrm{Al}+1 \%$ silicon $)$ metal lines. Insulating PECVD nitride is deposited, followed by platinum evaporation to define electrical recording sites. For back-side etching, a polymer coating Protek SR-25 is spun to protect the frontside features during DRIE probe release. This procedure avoids the large sidewall angle with $\mathrm{KOH}$ on $<100>$ wafers, and non-uniformities in $\mathrm{KOH}$ etch rates that allows $\mathrm{KOH}$ to leak to the frontside films. The new process has an improved yield near $90 \%$ versus $60 \%$ with $\mathrm{KOH}$. Lead Zirconate Titanate Oxide (PZT-4H) piezoelectric plates $\left(3.55 \times 1.25 \times 0.5 \mathrm{~mm}^{3}\right)$ are affixed to the zero-displacement nodes of the longitudinal mode shape. Based on finite element simulations, a longitudinal resonance frequency of $430 \mathrm{kHz}$ is expected, which is close to the impedance analyzer measurement on fabricated devices of $416 \mathrm{kHz}$.

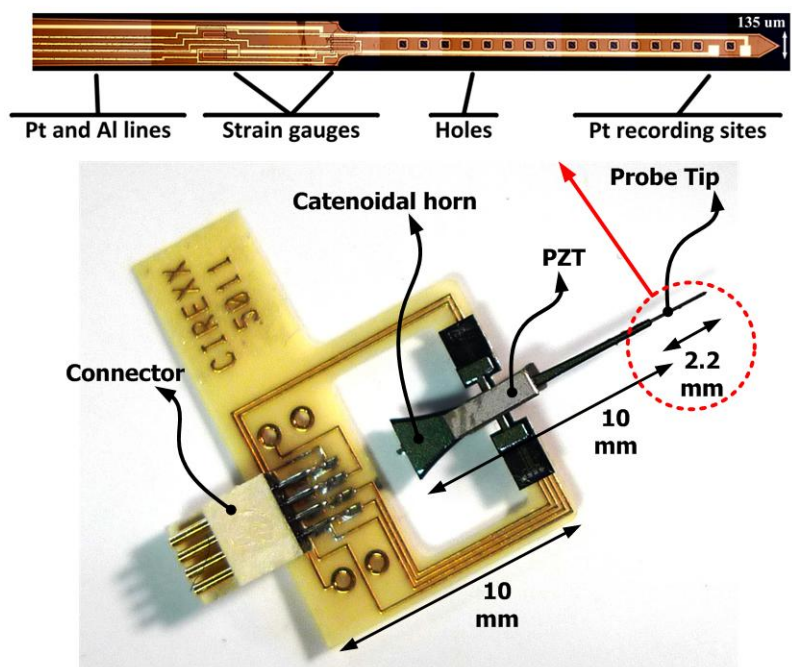

Figure 2: Miniature silicon horn with piezoelectric plates to drive the probe at its longitudinal resonance, and microphotograph of probe tip with integrated strain gauges and platinum recording sites
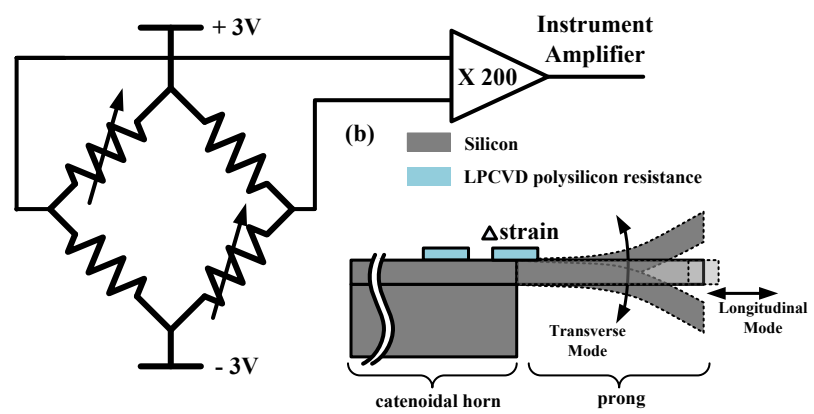

Figure 3: (a) Wheatstone bridge circuit configuration for recording insertion force and micromotion. (b) Two different modes (longitudinal/transverse) of probe tip will induce the strain signal

A prong off the main horn forms the neural probe. The prong consists of two electrical sites and polysilicon strain gauges at the interface of the horn and the prong for optimum strain measurement as shown in Fig. 3. As the probe tip bends, the resistances on the variable polyresistors change allowing monitoring of strain on the probe. We characterize this response using prong-probe dimensions of $2.2 \mathrm{~mm}$ length, $135 \mathrm{~m}$ width and $140 \mathrm{~m}$ thickness, resulting in a transverse mode displacement sensitivity of $43 \mathrm{mV} / \mathrm{m}$, and a longitudinal mode force sensitivity of $20 \mathrm{mV} / \mathrm{mN}$. Integrated holes $\left(35 \times 35 \mathrm{~m}^{2}\right)$ in the prong are fabricated to test the hypothesis that the holes may allow for better signal transduction and biocompatibility. The horn and probe is placed on a custom flexible printed circuit board (PCB) and interfaced to our custom miniature recording system.

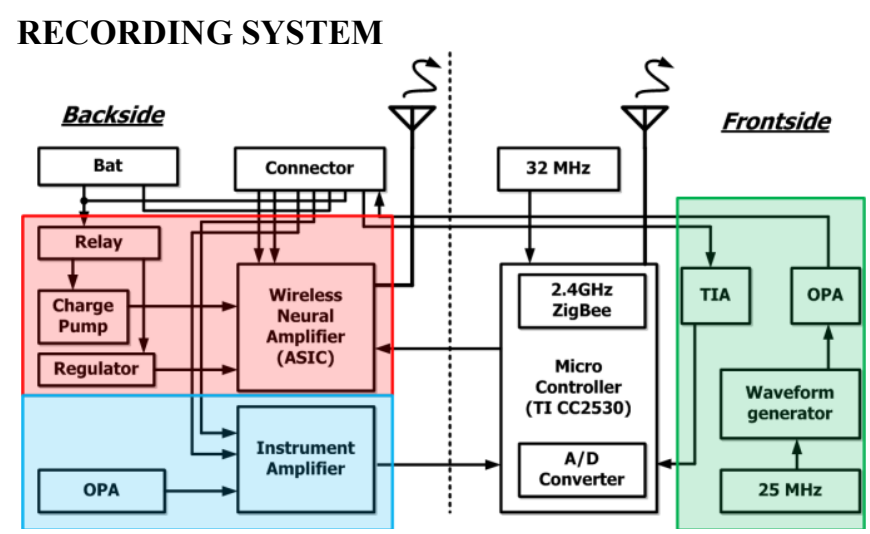

Figure 4: Control circuit block diagram. Different color correlated to the different functionalities: red- neural recording, blue insertion force and micromotion, and green - ultrasonic response.

The whole system block diagram is shown in Fig. 4. An integrated circuit was designed in Austrian Micro Systems (AMS) $0.35-\mathrm{m}$ process with four multiplexed neural amplifiers and a voltage control oscillator (VCO) as transmitter. A two-stage amplifier topology composed of differential stage followed by a common source stage resulted in a $1.25 \mathrm{~V}$ input refereed noise and $50 \mathrm{~dB}$ gain over $10 \mathrm{kHz}$ bandwidth. The VCO is designed using a differential ring oscillator topology to avoid external components. The telemetry frequency range is designed to be 82 to $84 \mathrm{MHz}$. An instrument amplifier (AD8223) with an operational amplifier (TI OPA890) is used to amplify the strain signal from the probe and send it to the analog-to-digital converter (ADC) in the microcontroller. A programmable waveform generator is controlled by the microcontroller to generate the desired frequency range of a sinusoid wave to actuate the probe and to locate the resonance frequency through a transimpedance amplifier (TIA) configured operational amplifier and send to the ADC. A microcontroller (Texas Instruments CC2530) is used to control these three different functions through a customized Lab-VIEW interface and to transmit them with a ZigBee RF interface. The system is built up on a four-layer two-sided PCB. A picture of the chronic recording system is shown in Fig. 5.

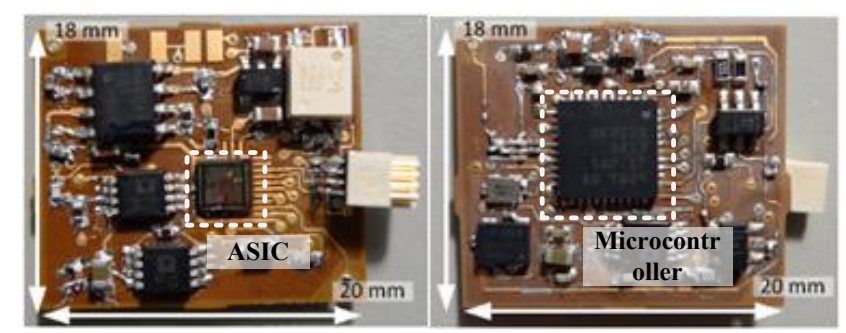

Figure 5: photograph of purposed chronic recording system 


\section{IN VIVO IMPLANTATION}

Preoperative care is administered using aseptic techniques for chronic animals. For chronic surgeries, instruments and materials are sterilized by autoclaving. Anesthesia is administered by $5 \%$ isoflurane (VetOne) and is maintained at $1.5-2 \%$ for the duration of the experiment.

At the beginning of the surgery, mice receive an injection of glycopyrrolate, an anticholinergic, intramuscularly at 0.002 $\mathrm{mg} / 100 \mathrm{~g}$ to assist in keeping the airways clear of fluid build-up and Ketoprofen $(5 \mathrm{mg} / \mathrm{kg})$ and Dexamethasone Sodium Phosphate $(0.2$ $\mathrm{mg} / \mathrm{kg}$ ) are administered subcutaneously. The mouse is secured in a stereotaxic frame by standard ear bars. After surgical preparation of the area on the head, bupivacaine $0.125 \%(\sim 0.1 \mathrm{ml})$ is injected subcutaneously above the skull. A short incision is made on top of the mouse's skull and the connective tissue is scraped away, exposing the bone.

a. Craniotomy - The dry skull is coated with a thin layer of cyanoacrylate adhesive (Vetbond). Three screws are placed in the skull to provide a framework for the dental cement to create a strong adhesion. A $5 \mathrm{~mm}$ diameter opening is drilled inside the chamber, leaving a bone flap in the center. After the bone flap is lifted off, the dura mater is kept moist with artificial cerebrospinal fluid (ACSF) and the chamber is filled with ACSF.

b. Insertion of electrode probe - The probe is secured and slowly lowered to the base of the craniotomy by a fine-precision motorized micromanipulator. The probe is gently inserted and advanced into the brain until it is approximately $200 \mu \mathrm{m}$ under the surface of the cortex. The probe is secured to the base of the skull with dental cement (Fig. 6).

c. Placement of glass coverslip - A glass coverslip is glued above the craniotomy site with cyanoacrylate and dental cement. The skin is closed around the margins of the cranial window with cyanoacrylate.

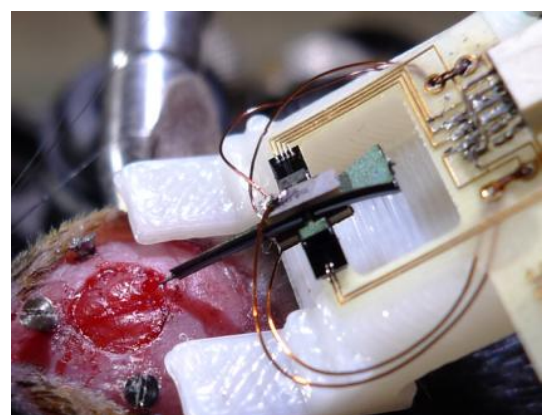

Figure 6: photograph of inserted probe in mouse brain

\section{IN VIVO EXPERIMENTAL RESULTS}

The neural probes are tested for biological efficacy by inserting them into the motor sensory cortex region of genetically modified C57BL/6 mice brains. The microglia cells were identified with green fluorescent protein (GFP). The ambulatory experiment setup is shown in Fig. 7.

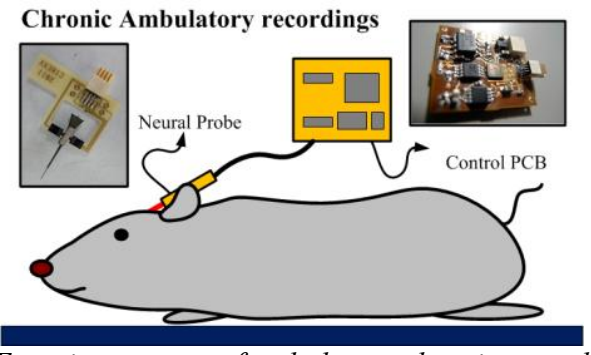

Figure 7. Experiment setup of ambulatory chronic recording.

\section{Insertion force reduction and net stress reduction}

PZT actuation is used to actuate probes during insertion while measuring the force required using a load cell. The force goes to a maximum as the probe punctures the tissue, after which the viscous drag of the tissue presents a constant force during insertion. This force can be used to monitor excessive stress caused by insertion. Different driving voltage verses the insertion forces were characterized with a $2 \%$ agar gel as brain tissue phantom (Fig. $8 \mathrm{a}$ ). The insertion force was reduced by a factor of 2.3, indicating that even at $5-\mathrm{Vpp}$ drive, the probes ultrasonically modify tissue insertion. Ultrasonic insertion causes the tissue-probe interface to have less net-stress by a factor of 1.5 , due to the ultrasonic cutting leading to less average stress and providing stress relief (Fig. 8b). The histology slices shown in Fig. 9 indicate that the ultrasonic insertion results in a smoother tissue boundary at the insertion site. The result is from the same animal with insertion into two different locations.
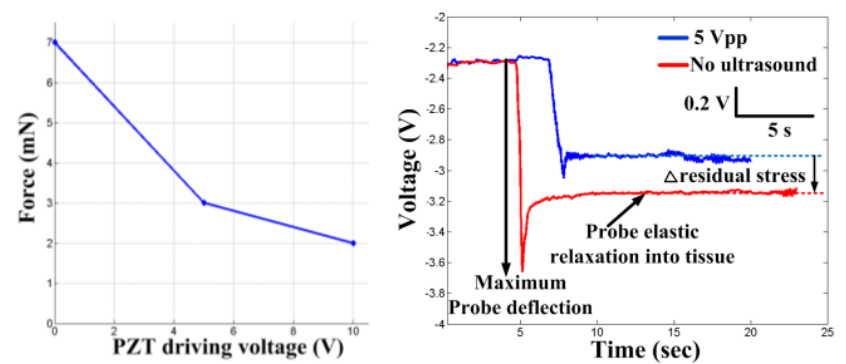

Figure 8: (a) Insertion force verse PZT driving voltage curve. (b) In vivo strain-gauge measured insertion force with no ultrasound and with 5-Vpp PZT driving voltage. Less probe deflection and residual stress by a factor of 1.5 with PZT driving was observed.
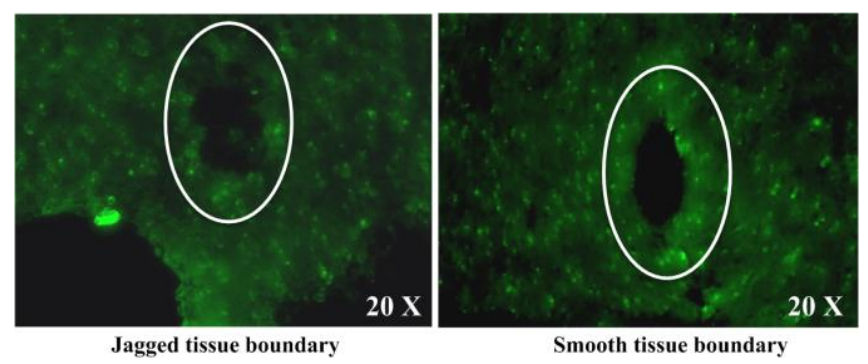

Figure 9: Histology result from the same mouse, without (left) and with (right) ultrasonic actuated insertion.

\section{Long term mechanical coupling to tissue}

The AC electro-mechanical impedance of the ultrasonic transducer at a probe longitudinal mode resonance frequency, long term mechanical coupling to tissue can be assessed. The quality factor of the resonance is indicative of the energy loss into the tissue. The measured mechanical impedance spectrum for the probe in air and after insertion into the mouse brain (Fig. 10) shows a peak broadening and a decrease in quality factor resulting from probe damping. The resonance curve shifts $3.9 \mathrm{kHz}$ with probe insertion, with peak broadening and a decreased quality factor from 34.07 to 26.72 (90\% of peak voltage) (Table 1).

Table 1: Resonance frequency, bandwidth and quality factor of the probe between air and brain tissue

\begin{tabular}{|l|c|c|}
\hline Unit & Air & Brain Tissue \\
\hline Resonance Frequency (kHz) & 415.7 & 419.6 \\
\hline Bandwidth (kHz) & 12.2 & 15.7 \\
\hline Quality Factor & 34.07 & 26.72 \\
\hline
\end{tabular}




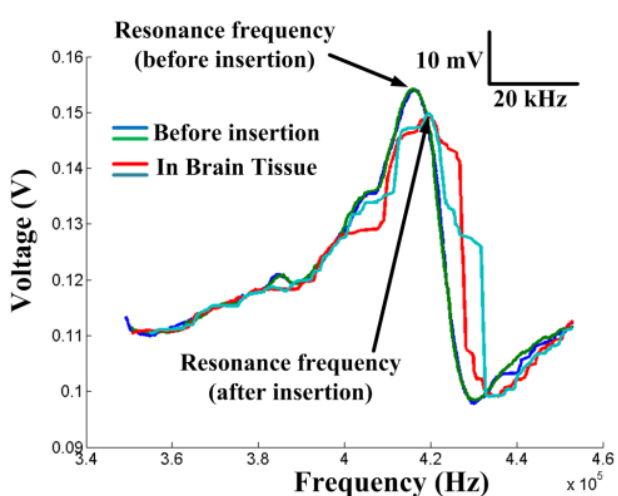

Figure 10: Measured mechanical impedance spectrum in air and in situ brain tissue

\section{Micromotion movement}

The strain-gauges can measure the strain caused by mouse motion and transmit wirelessly after insertion. We measured mouse breathing and heart rates as mechanical forces applied biologically to the mouse (Fig. 11). Studies have shown that blood flow and respiration can induce micromotion of the brain during craniotomies [10]. Our measurements indicate approximately 3- m movement induced by breathing inside the brain.

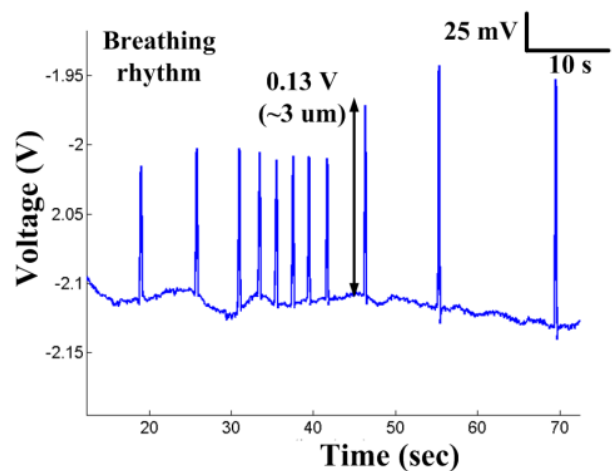

Figure 11: Strain-gauge measured breathing induced micromotion inside mouse brain.

\section{Chronic neural electrical signal measurement}

Ambulatory chronic recording after implanting the electrode for 10 days is shown in Fig. 12. The spike rates and electrode impedance from the mouse brain can be monitored over time to determine aging of the neural interface.

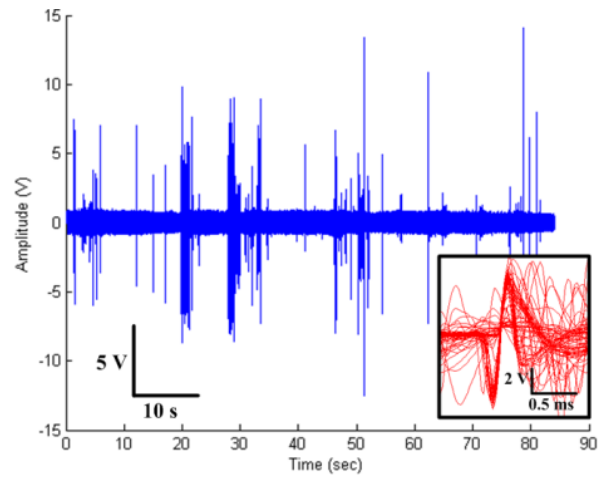

Figure 12: Chronic neural recordings from probe Pt recording sites after 10 days of implantation.

\section{CONCLUSION}

In this study, we have demonstrated a microfabricated neural probe with the capability to measure and alter the mechanical environment inside the tissue. In addition, the miniature recording system can record the signal simultaneously and chronically. We have demonstrated insertion force reduction, tissue-probe stress reduction, measurement of micromotion, and the probe resonance measurement. Future work and challenges include miniaturizing the system in an ASIC, and collecting more chronic recording data to further identify key variables that improve neural probe reliability.

\section{ACKNOWLEDGMENT}

This work was sponsored by the Defense Advanced Research Projects Agency (DARPA) MTO under the auspices of Dr. Jack Judy through the Space and Naval Warfare Systems Center, Pacific Grant/Contract No. N66001-11-1-4011. We would like to thank the Cornell Nanofabrication Facility (CNF) at Cornell University for fabrication of the probe.

\section{REFERENCES}

[1] M.A. Lebedev, J.M. Carmena, J.E. O'Doherty, M.Zacksenhouse, C.S. Henriquez, J.C. Principe, and M.A.L. Nicolelis, "Cortical ensemble adaptation to represent velocity of an artificial actuator controlled by a Brain-Machine Interface," J. Neuroscience, 25, 19 (2005), pp. 4681-93

[2] V.S. Polikov, W.M. Reichart, and P.A. Tresco, "Response of brain tissue to chronically implanted neural electrodes," J. Neurosci. Methods, 148, 1 (2005), pp. 1-18.

[3] D.J. Edell, V.V. Toi, V.M. McNeil, and L.D. Clark, "Factors influencing the biocompatibility of insertable silicon microshafts in cerebral cortex," IEEE Trans. Biomed. Eng., 39, 6 (1992), pp. 635-43.

[4] P.J. Rousche and R.A. Normann, "A system for impact insertion of a 100 electrode array into cortical tissue," in Proc. Int. IEEE EMBS Conference. (1990), pp. 494-95.

[5] D.-H. Kim, et al., "Dissolvable films of silk fibroin for ultrathin conformal bio-integrated electronics," Nat. Mater., 9, 6 (2010), pp. 511-17.

[6] K.A. Ludwig, N.B. Langhais, M.D. Joseph, S.M. Richardson-Burns, J.L. Hendricks, and D.R. Kipke, "Poly(3,4-ethylenedioxythiophene) (pedot) polymer coatings facilitate smaller neural recording electrodes," J. Neural Eng., 8, 1 (2011), pp. 014001.

[7] X.T. Cui and D.D. Zhou, "Poly(3,4-ethylenedioxythiophene) for chronic neural stimulation," IEEE Trans. Neural Syst. and Rehabil. Eng., 15, 4 (2007), pp. 502-08.

[8] K.A. Ludwig, J.D. Uram, J. Yang, D.C. Martin, and D.R. Kipke, "Chronic neural recordings using silicon microelectrode arrays electrochemically deposited with a poly(3,4-ethylenedioxythiophene) (pedot) film," J. Neural Eng., 3, 1 (2006), pp. 59-70.

[9] C.J. Shen, R.F. Gilmour, Jr. and A. Lal, "Silicon ultrasonic microprobes with collocated and concurrent strain and bio-electrical potential wireless transmission," Proc. MEMS Jan. (2011), pp.972-75.

[10] X. Liu, D.B. McCreery, R.R. Carter, L.A. Bullara, T.G. Yuen, and W.F. Agnew, "Stability of the interface between neural tissue and chronically implanted intrcortical microelectrodes," IEEE Trans. Rehabil. Eng. , 7, 3 (1999), pp. 315-326.

\section{CONTACT}

*P.C. Chen, tel: +1-607-793-3300; pc445@cornell.edu 